\title{
AC 2007-2319: DATA ACQUISITION AND COMPUTER SIMULATION INTEGRATED EXPERIMENT FOR AN UNDERGRADUATE MACHINE DYNAMICS LABORATORY
}

\section{Petru-Aurelian Simionescu, University of Tulsa}

Petru-Aurelian Simionescu is currently an Assistant Professor of Mechanical Engineering at The University of Tulsa. His teaching and research interests are in the areas of Dynamics, Vibrations, Optimal design of mechanical systems, Mechanisms and Robotics, CAD and Computer Graphics. $\mathrm{He}$ is on leave to the University of Alabama at Birmingham.

Jeremy S. Daily, University of Tulsa

Jeremy S. Daily is an Assistant Professor of Mechanical Engineering at The University of Tulsa. His teaching and research interests are in the areas of Computer Aided Engineering, Solid Mechanics, Vehicle Dynamics and Traffic Crash Reconstruction.

\section{John R. Shadley, University of Tulsa}

John R. Shadley is Emeritus Professor of Mechanical Engineering at The University of Tulsa. He taught solid mechanics courses and laboratory classes at the University of Tulsa, and was engaged in research projects involving solid mechanics and materials. 


\title{
Data Acquisition and Computer Simulation Integrated Experiment for an Undergraduate Machine Dynamics Laboratory
}

\begin{abstract}
A cam-follower mechanism experiment is described that involves the calibration of several mechanical transducers and computerized data acquisition. Laboratory measurements are followed by modeling the experiment using multi-body simulation software (VisualNastran 4D and Working Model 2D). Students compare their simulations with the measured results and learn about the benefits of integrating physical experiments with virtual experiments. Their confidence in the results obtained through simulation, as well as their preference for performing either one of both type of experiments are surveyed.
\end{abstract}

\section{Introduction}

This paper describes an experiment that has been developed for the Machine Dynamics course in the University of Tulsa's Mechanical Engineering Department. This four-credit hour, junior level course contains six laboratory experiments as follows:

- Operating point of a battery powered sweeper - apparatus was custom made;

- Free and forced vibration analyses of a single degree-of-freedom system using a TM16 apparatus from TQ Education and Training Ltd. ${ }^{1}$;

- Static and dynamic balancing of rotating masses using a TM102 apparatus from TQ Education and Training Ltd. ${ }^{2}$;

- Flexible rotor dynamics experiment - apparatus was custom made as senior-design project;

- Experimental analysis of a cam follower mechanism - apparatus was custom made as a senior-design project ${ }^{3}$ and retrofitted to work with LabVIEW $^{4}$ data acquisition software.
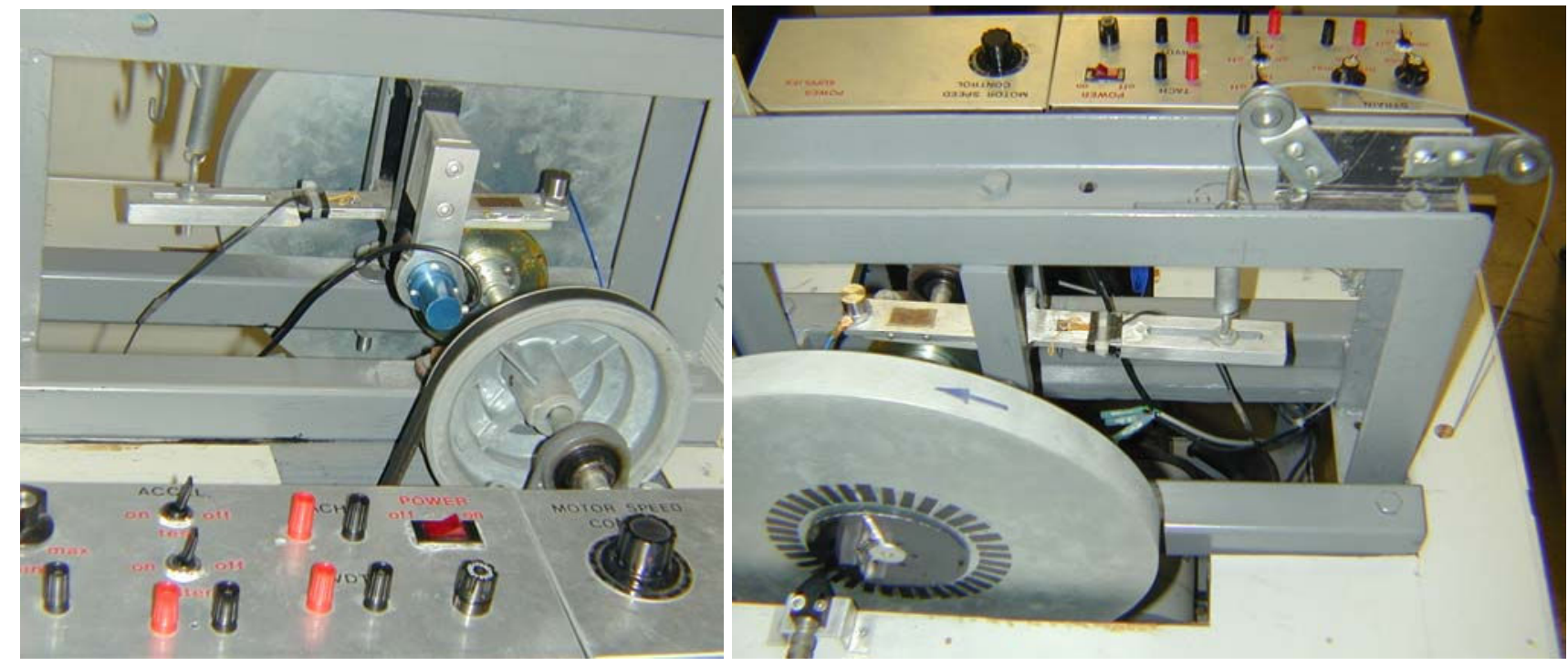

Fig. 1: Cam-follower experimental apparatus 
These labs were recently found to be major contributors to the ABET criteria outcomes $\boldsymbol{b}$ (an ability to design and conduct experiments, as well as to analyze and interpret data) and $\boldsymbol{k}$ (an ability to use techniques, skills and modern engineering tools for engineering practice).

The last of these experiments is also the most complex, involving calibrating several transducers and acquiring data using the computer. As part of the laboratory assignment, students model the cam-follower mechanism using multi-body simulation software VisualNastran $4 \mathrm{D}^{5}$ or Working Model $2 \mathrm{D}^{6}$ and compare simulation and measurement results. They work in teams of 3-4 members and document their lab experience and results in a formal technical report.

\section{Cam-Follower Experiment Description}

The purpose of the cam-follower experiment is to determine the displacement, velocity, and acceleration of the follower of an eccentric cam mechanism. In the process students learn about the calibration and operation of several transducers that are used in the

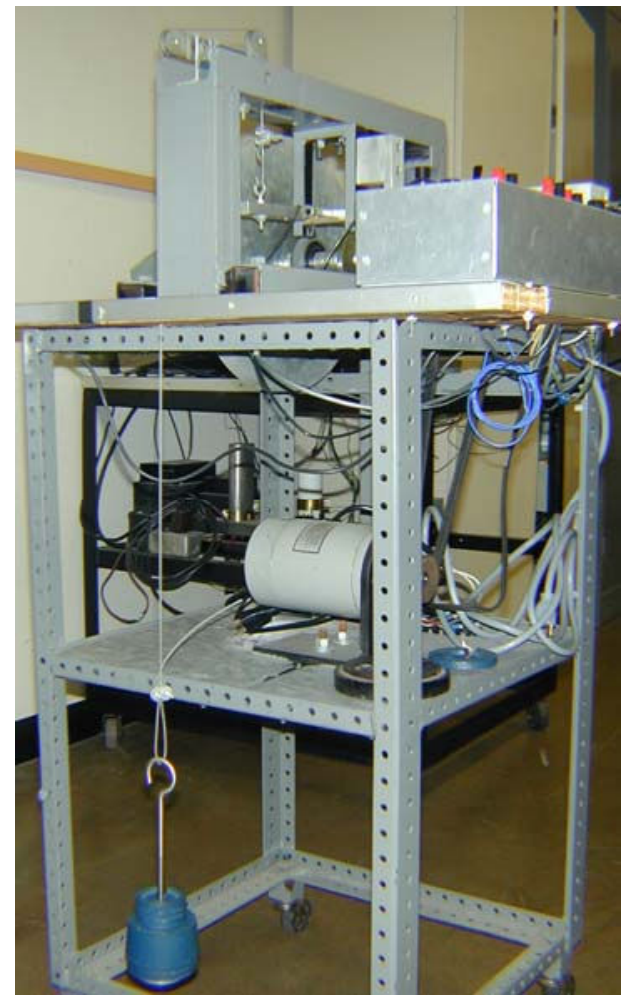

Fig. 2: Strain gage calibration experiment: incremental optical encoder, rotary variable differential transformer (RVDT), straingage based force transducer, and piezoelectric accelerometer. They also study the effect of sample rate upon measured signals, and learn how the Fast Fourier Transform (FFT) can be used to determine the fundamental frequency of a periodic signal.

Table 1. Equipment List

\begin{tabular}{|l|l|l|l|l|}
\hline & Item & Manufacturer & Model/Version & Cost \\
\hline 1 & Strain Gages & Vishay Micro-Measurements & CEA-13-240UZ-120 & $\$ 10$ \\
\hline 2 & RVDT & Schaevitz Sensors & R30D & $\$ 170$ \\
\hline 3 & Photoelectric Probe & Brüel \& Kjær & MM0012 & $\$ 68$ \\
\hline 4 & Piezoelectric Accelerometer & Brüel \& Kjær & 4368 & $\$ 350$ \\
\hline 5 & PCI Bus Data Acquisition Board & Keithley Instruments Inc. & KPCI-3108 & $\$ 550$ \\
\hline 6 & Screw Terminal Connector & Keithley Instruments Inc. & STP-36 & $\$ 77$ \\
\hline 7 & Sensor Signal Conditioner & PCB Piezotronics Inc. & 482 A21 & $\$ 280$ \\
\hline 8 & LabVIEW Software & National Instruments & Ver. 6.1 & - \\
\hline 9 & Variable Speed DC Motor & Indiana General & A35D 20023-01 & $\$ 185$ \\
\hline
\end{tabular}

The mechanical part of the experiment (Fig. 1) is a cam - oscillating follower mechanism. The cam is a disk of radius 1.75 inches and eccentricity 0.26 inches, while the distance between the axis of the cam and that of the follower is 3.35 inches. A tension spring maintains contact between the follower and the cam surface. An adjustable speed motor and a belt-pulley transmission drive a horizontal shaft supporting the cam and a large flywheel. On the side of the flywheel an optical encoder is mounted and used as cam-shaft tachometer. 
The follower angle is measured using an RVDT connected to the rotating axle of the follower, while the stationary part of the RVDT sits in a circular mount attached to the frame of the machine. Two metal foil strain gages are bonded on the surface of the follower and are used to measure the tension of the spring. A uniaxial piezoelectric accelerometer is attached to the tip of the follower. A list of the electrical components used in the experiment together with their cost is available in Table 1.

The computer data acquisition system used to record the signals from the transducers consists of a PCI bus data acquisition board, screw terminal connector, signal condition modules, and LabVIEW software. While the students do not write the data acquisition code or design the user interface, they are shown the underlying LabVIEW wiring diagram and are required to explain how the data acquisition system works.
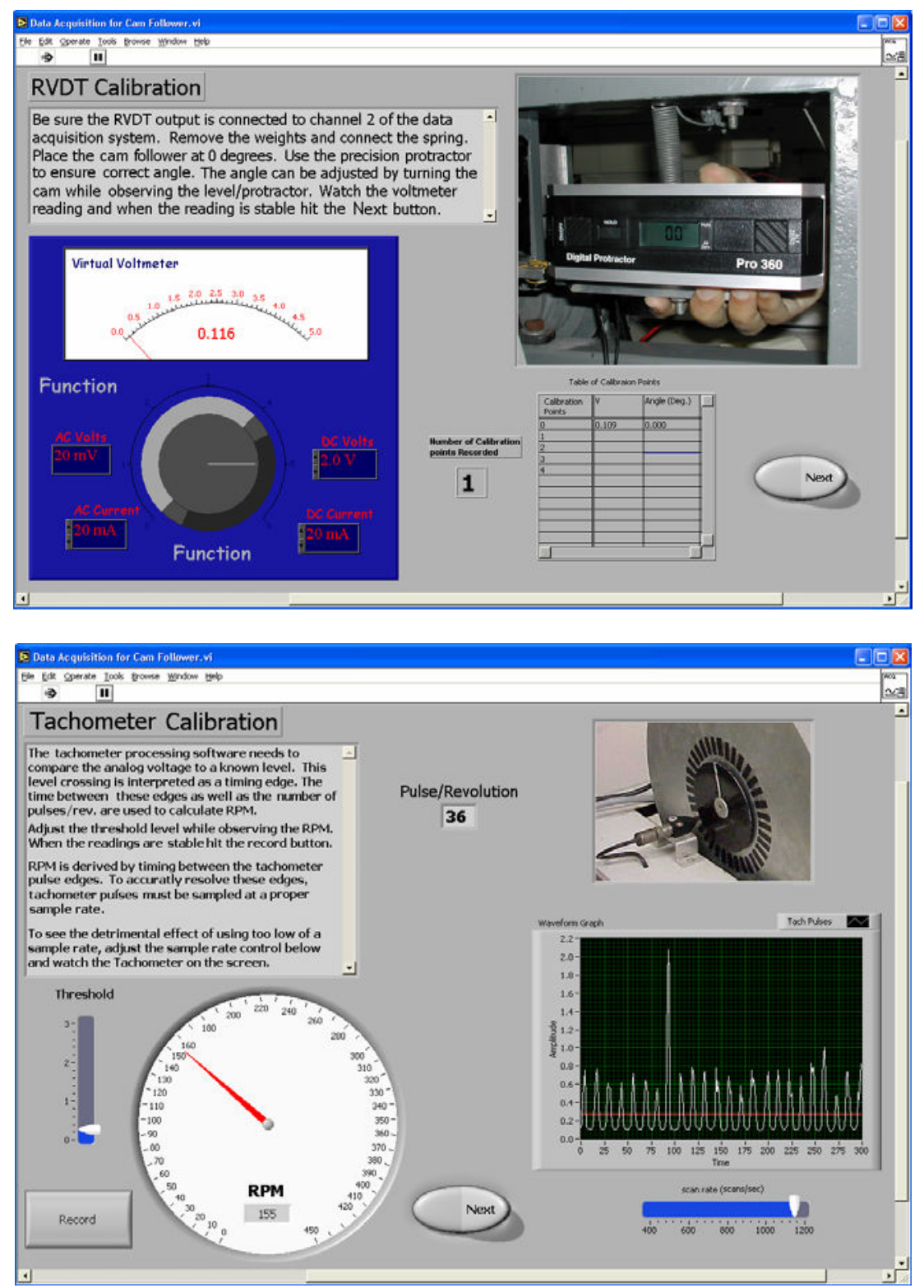

Fig. 3: LabVIEW front panels for calibration of the RVDT and tachometer calibration 


\section{Calibration and Data Acquisition}

The electrical resistance strain gages are bonded on the upper and lower surface of the follower, close to its axis of rotation where the bending moment is higher. They are connected into a Wheatstone bridge circuit that produces an output voltage proportional to the amount of deformation of the gage wires. When the cam lifts the follower, it bends proportional to the load applied by the spring, causing the upper gage to contract and the lower gage to elongate. To calibrate the strain gages as a force transducer, predetermined loads are applied at the spring hook using a cable and pulley system (see Fig. 2).

Calibration of the $\boldsymbol{R} \boldsymbol{V D T}$ is accomplished by inducing known angular rotations to the follower using a digital protractor, and measuring the corresponding voltages. A number of four calibration points between zero and the maximum lift of the follower are manually fixed and recorded (Fig. 3-up).

The makeshift tachometer used to determine the speed of the cam consists of an optical encoder with the photoemitter-detector arranged in retroreflective-mode. For every rotation, the detector receives 36 pulses, of which one is more intense and corresponds to the follower in its upper most position. Calibration of the tachometer requires adjusting the threshold voltage that will

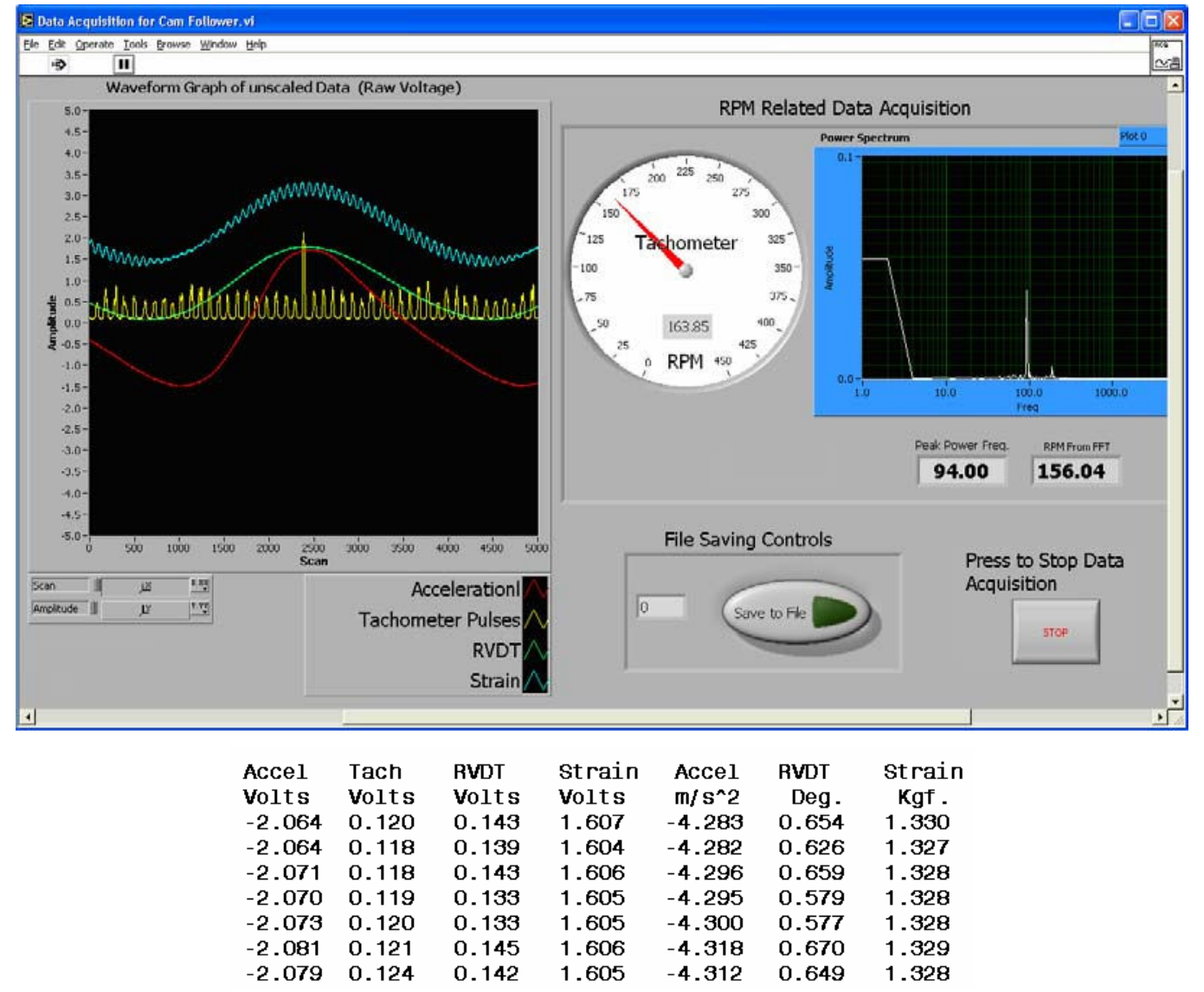

Fig. 4: LabVIEW data acquisition front panel (above), and output data file structure (below). 
allow the software to resolve the edges between these pulses (Fig. 3-down). With this same occasion students vary the rate at which the tachometer signal is sampled and observe the effect of the aliasing phenomenon, which occurs when the sampling rate is too low.

No calibration of the piezoelectric accelerometer is performed by the students. It is only explained to them that the manufacturer's charge sensitivity data were embedded in the LabVIEW program. They are also made aware that the axis of maximum sensitivity of the accelerometer is perpendicular to the upper surface of the follower, and that the center of its seismic mass is $10 \mathrm{~mm}$ from the base.

After calibration is complete, the students adjust the data acquisition rate, enter the number of samples they want collected, and record these samples to the computer. The LabVIEW program employs two methods for calculating the RPM of the cam-shaft (see Fig. 4-above). One method
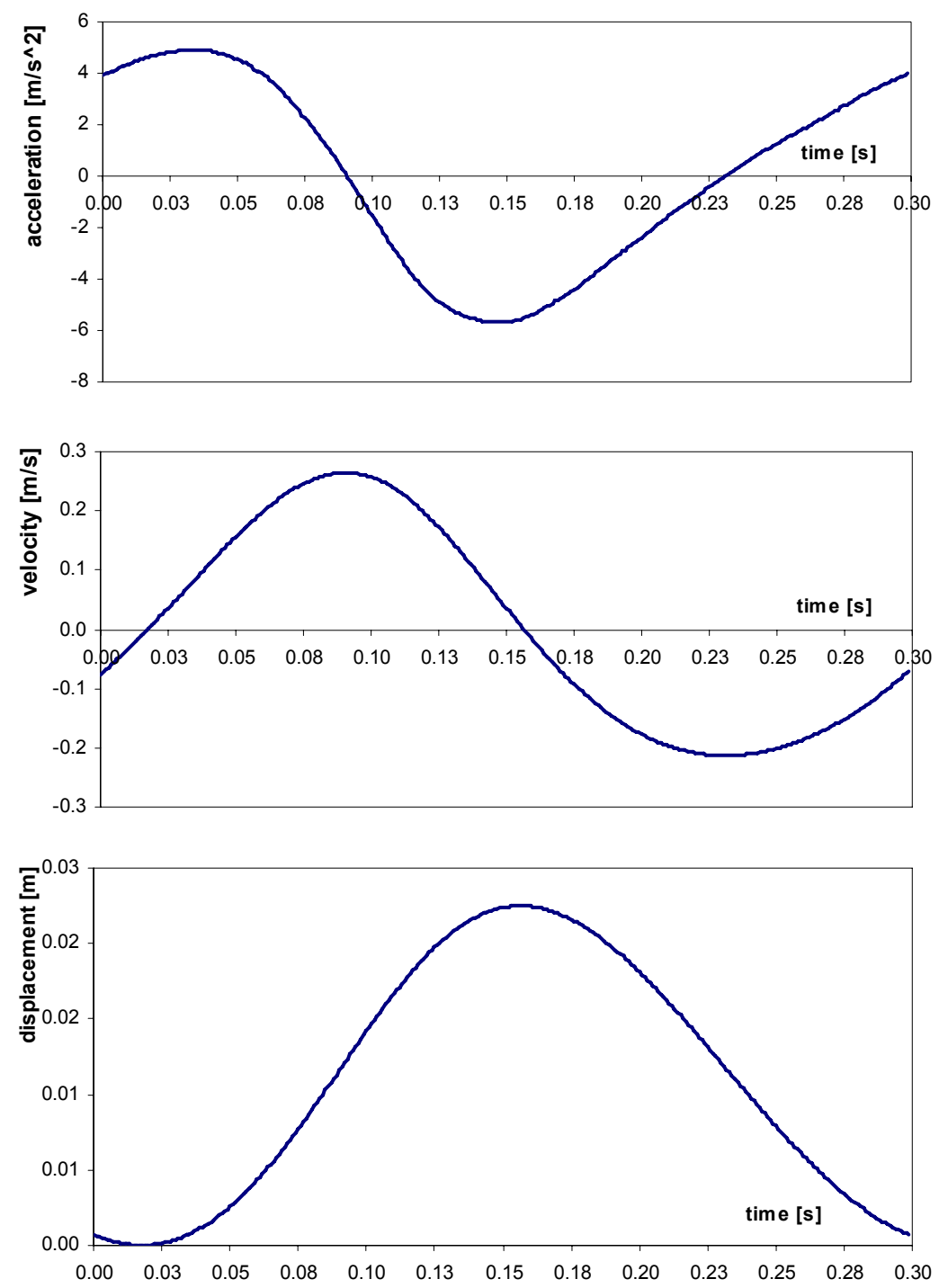

Fig. 5: Plots of the acceleration (from direct measurement), velocity and displacement (obtained through numerical integration) of the tip of the follower. 


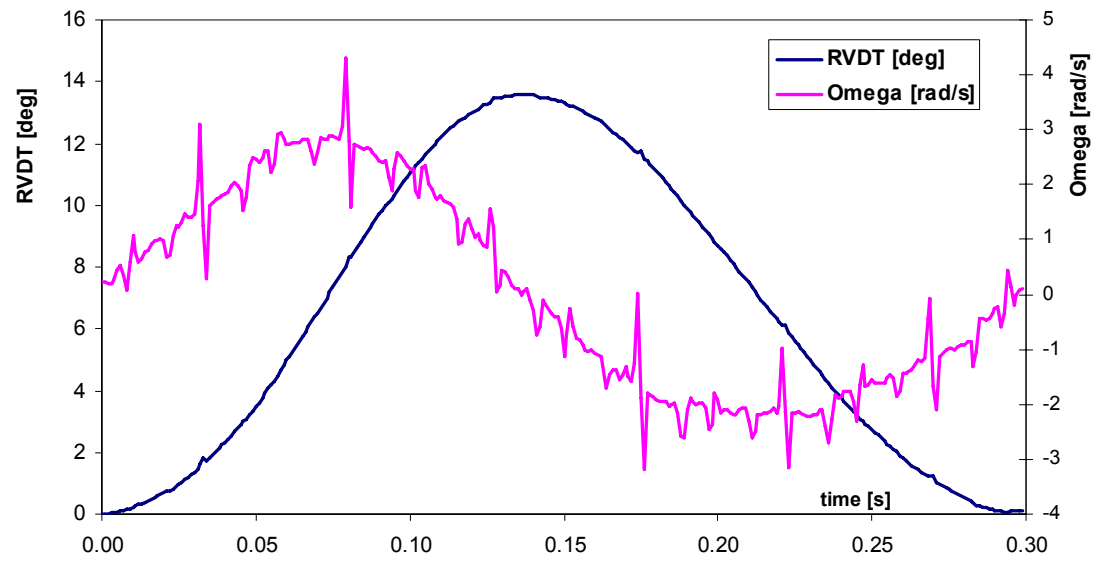

Fig. 6: Displacement of the follower (from direct measurement) and its angular velocity (obtained through numerical differentiation).

uses the tachometer-pulse count and the other method uses the fundamental frequency of the tachometer voltage extracted through an FFT analysis. Once this frequency $f$ in $\mathrm{Hz}$ is available, the RPM of the cam can be easily calculated as:

$$
\mathrm{RPM}=60 \cdot f / 36
$$

where 36 is the number of pulses per cam-shaft turn (i.e. equal to the number of shiny stripes of the optical encoder disk) received on the tachometer channel.

In addition to the voltages from the strain gages, RVDT, tachometer and acceleration channels, the LabVIEW program saves to the hard drive the acceleration, follower displacement, and spring force calculated in engineering units (see Fig. 4 sample data).

\section{Data Interpretation}

The students use MS Excel to select one motion cycle of the mechanism from the acquired data (same initial and final follower angle as measured by the RVDT). Since it is not readily available, they have to generate a column of time values using the scan rate information. Then they plot as functions of time the tachometer pulses, follower displacement, linear acceleration of the tip of the follower and spring force. Part of the lab assignment is to generate the velocity and displacement of the tip of the follower by integrating the acceleration signal using the Trapezoidal Rule. For this particular system with periodic motion, the initial velocity should be such that for one motion cycle, the first and last values of the displacement of the follower coincide (Fig. 5). This is done conveniently by adjusting the initial velocity using the Goal Seek tool in MS Excel.

Finally, to show that the noise inherent to any experimental data acquisition are accentuated when derivatives are calculated numerically, the students plot the angular velocity of the follower calculate by taking the finite-difference derivative of the follower displacement (Fig. 6). 


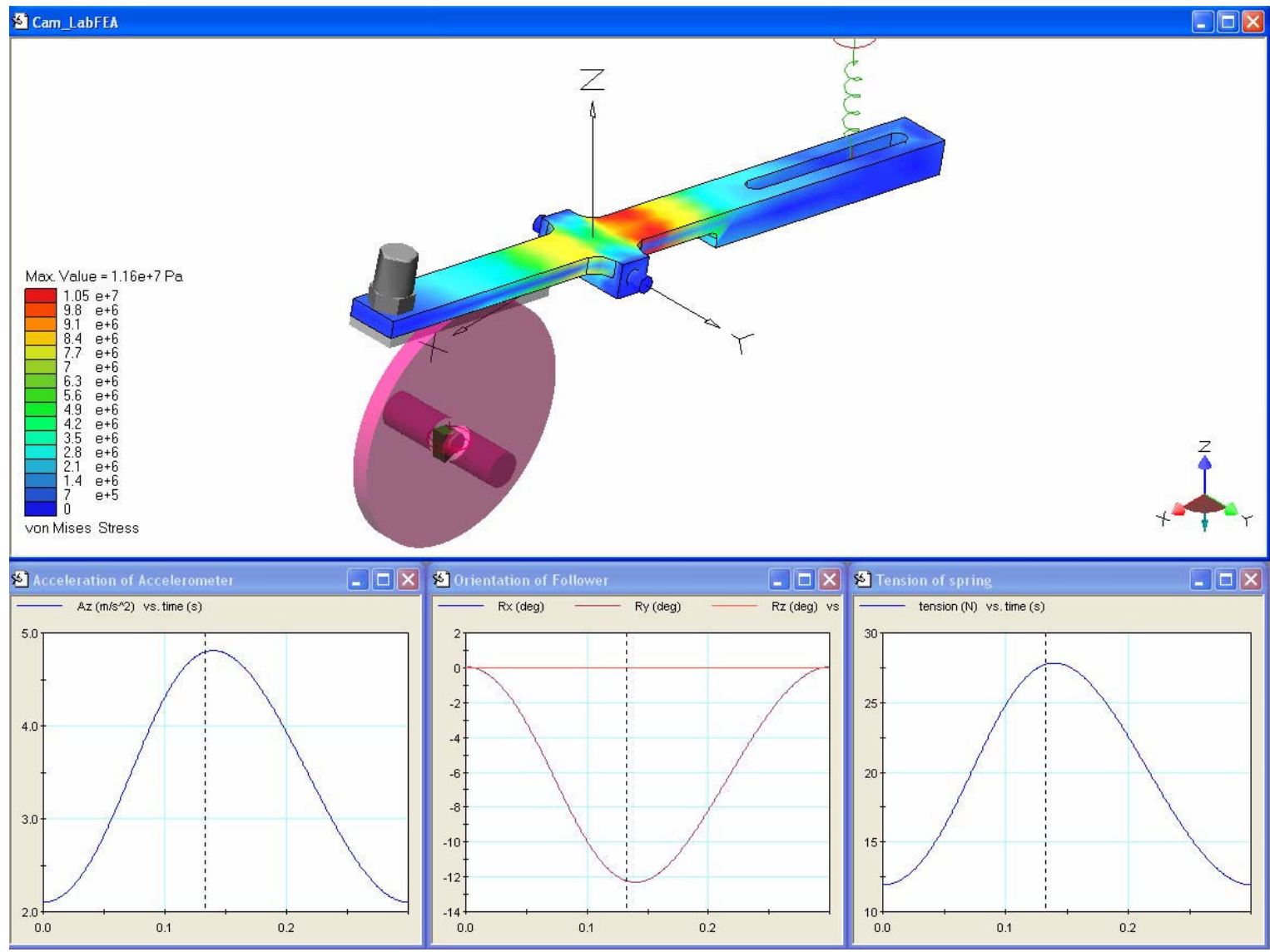

Fig. 7: Visual Nastran 4D simulation of the cam-follower experiment. With little effort students could perform FEA of the follower, and observe that the most stressed areas are those where the strain gages are bonded.

\section{Muti-body Simulation}

As part of their laboratory experience, the students simulate the cam-follower mechanism using a commercially available multi-body software, when driven at the same constant RPM as the real apparatus. Until recently Visual Nastran 4D from MSC.Software Corporation was employed in this assignment (Fig. 7). This is a user-friendly, powerful software program with some Finite Element Analysis capabilities that students enjoyed using.

It is apparent that MSC.Software is no longer supporting Visual Nastran 4D (although no official statement has been made yet), and beginning in 2005 Working Model 2D is used instead (Fig. 8). The students model the mechanism components and assemble them using the necessary constraints. Any relevant dimension and joint locations are extracted from the mechanical drawing of the experimental apparatus ${ }^{3}$ or through direct measurement. The rate $k$ of the elastic spring of the follower is calculated using the following formula ${ }^{7}$ :

$$
k=\frac{d^{4} \cdot \mathrm{G}}{8 \cdot D^{3} \cdot \mathrm{N}}
$$

where $d$ is the diameter of the wire, $D$ is the mean diameter of the coil, $\mathrm{N}$ is the number of active coils and $\mathrm{G}$ is the shear modulus of the spring material. 

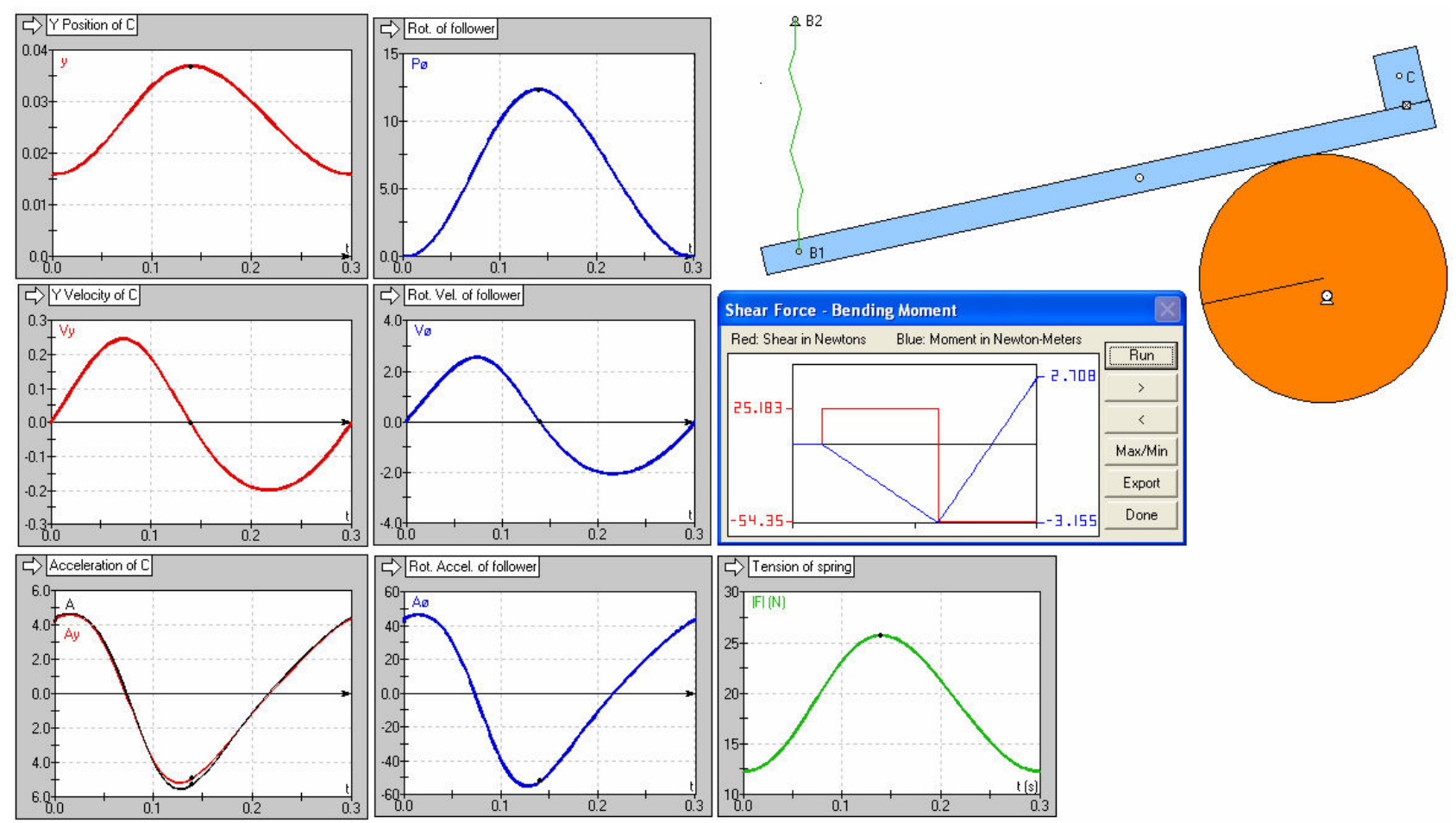

Fig. 8: Working Model 2D simulation of the cam-follower experiment. The acceleration read by the accelerometer was approximated as the y component of the total acceleration of point $\mathrm{C}$, and as the tangential acceleration of point C. In addition, the students are requested to exercise with the Shear Force Bending Moment script available in Working Model 2D.

Having the measurement results available, the students are able to check for errors in their model and to adjust the free length of the spring until the same spring tension is obtained in Working Model 2D as in the LabVIEW measurements. They are left to discover that the acceleration measured experimentally is not exactly the tangential acceleration of the center of the accelerometer (point $\mathrm{C}$ in Fig. 8), but rather the projection of the total acceleration of this point $\mathrm{C}$ to the principal axis of the accelerometer. As part of their lab report students are required to explain the reason for any differences between the measured results and their simulations, and the one just mentioned is the most noticeable.

\section{Student Assessment}

After the students turned in their laboratory reports (two weeks after performing the experiments), a short survey was given in order to assess how this laboratory exercise was received. On a six point Likert scale students indicate if they strongly disagree, disagree, slightly disagree, slightly agree, agree, or strongly agree with seven statements. A number from 1 (for strongly disagree) to 6 (for strongly agree) was assigned to each selection. A total of 14 students responded to the survey and the results are summarized in Table 2 below. 
Table 2. Student Survey Results

\begin{tabular}{|l|l|c|}
\hline & Question: & $\begin{array}{l}\text { Average } \\
\text { response: }\end{array}$ \\
\hline 1 & $\begin{array}{l}\text { The experiment did a good job of familiarizing me with typical instrument calibration } \\
\text { procedures. }\end{array}$ & 4.00 \\
\hline 2 & $\begin{array}{l}\text { The laboratory did a good job of familiarizing me with computerized data acquisition and } \\
\text { LabVIEW. }\end{array}$ & $\begin{array}{l}\text { The laboratory did a good job of familiarizing me with the operation of a rotary variable } \\
\text { differential transformers (RVDT), piezoelectric accelerometers, and of strain-gauge force } \\
\text { transducers. }\end{array}$ \\
\hline 4 & $\begin{array}{l}\text { As an educational experience, computer simulation complemented well the hardware } \\
\text { experiment. }\end{array}$ & 4.86 \\
\hline 5 & $\begin{array}{l}\text { The experiment and subsequent simulation caused me to think critically about the } \\
\text { differences between the measured and the simulated results. }\end{array}$ & 4.57 \\
\hline 6 & Overall my preference inclines more towards the hardware experiment. & 4.07 \\
\hline 7 & Overall my preference inclines more towards the computer simulation. & 4.79 \\
\hline
\end{tabular}

\section{Conclusions}

The cam-follower lab experiment and multi-body simulation thereafter offer a complex and rewarding educational experience to mechanical engineering students. They see several transducers in use (strain gages, RVDT, optical-encoder, accelerometer), calibrate three of these transducers, and perform computerized data acquisition. In the process students experiment with aliasing in data acquisition and learn how Fast Fourier Transforms can be used to extract the fundamental frequency of a periodic signal. Students then analyze the acquired data, perform numerical integration and differentiation while observing the limitations of the latter. Finally, they use computer simulation software (originally MSC.VisualNastran 4D and more recently Working Model 2D) to model the experiment and plot the same parameters as in the physical experiment. This gives them the opportunity to better understand the meaning of the measured data, and provides them with an optimum learning experience that combines hands on experience with software simulation, found by many scholars in the past to be very effective ${ }^{8,9}$.

Although it was suggested ${ }^{10,11}$ that physical lab experiment can be totally replaced with virtual experiments and computer simulation, it is concluded here that a rich learning experience can be created with fewer but more involved laboratory experiments. The use of modern simulation and analysis software should be used to augment and reinforce the phenomena demonstrated with the physical experiment. This approach agrees with our students' preference and satisfies the desire to have more "hands-on" experiences as an engineering undergraduate.

The lab experiment described in this paper can be relatively inexpensively built as a senior design project in multidisciplinary teams from both Mechanical Engineering and Electrical Engineering majors. Many of the components can be reused from older pieces of equipment, or purchased at a fraction of their catalog price from online auctions. 


\section{Bibliography}

1. TM16 Universal Vibration Apparatus, TQ Education and Training Ltd., Nottingham, England, http://www.tq.com/product/index.asp?pid=tm16

2. TM102 Universal Vibration Apparatus, TQ Education and Training Ltd., Nottingham, UK, http://www.tq.com/product/index.asp?pid=tm102

3. Hanson, B.A. "Computerization of a Cam Follower Experiment using Data Acquisition System" The University of Tulsa, Mechanical Engineering Department, Senior Design Project, (advisor J. R. Shadley), 1989.

4. LabVIEW, National Instruments Corporation, Austin, TX, http://www.ni.com/labview/

5. Visual Nastran 4D, MSC Software Corporation, Santa Ana, CA, http://www.mscsoftware.com/support/prod\%5Fsupport/4d/

6. Working Model 2D, Design Simulation Technologies, Inc., Canton, MI, http://www.designsimulation.com/WM2D/index.php

7. Juvinal R.C. and Marshek K.M., "Fundamentals of Machine Component Design," Wiley, 2006.

8. Reamon, D.T. and Sheppard S.D., "The Role of Simulation Software in an Ideal Learning Environment," Proc. of the 1997 ASME Design Engineering Technical Conferences, Sept. 14-17, 1997, Sacramento, CA.

9. Knight, C.V. and McDonald G.H., "Attributes of a Modern Mechanical Engineering Laboratory," Proc. of the 2005 ASEE Annual Conference \& Exposition, June 12-15, 2005, Portland, OR.

10. Hannigan T., Koenig K., Austin V. and Okoro E., "Shelving the Hardware: Developing Virtual Laboratory Experiments," Proc. of the 2005 ASEE Annual Conference \& Exposition.

11. Helmer W.A, "Some New Design Techniques for Introductory Engineering Courses," ICEE 1997 International Conference on Engineering Education - Progress Through Partnerships, August 13-15, 1997, Chicago, IL. 